PROCEEDINGS OF THE

AMERICAN MATHEMATICAL SOCIETY

Volume 128, Number 8, Pages 2509-2511

S 0002-9939(00)05830-5

Article electronically published on April 11, 2000

\title{
WHEN PRODUCTS OF SELFADJOINTS ARE NORMAL
}

\author{
E. ALBRECHT AND P. G. SPAIN \\ (Communicated by David R. Larson)
}

\begin{abstract}
Suppose that $h, k \in \mathcal{L}(\mathcal{H})$ are two selfadjoint bounded operators on a Hilbert space $\mathcal{H}$. It is elementary to show that $h k$ is selfadjoint precisely when $h k=k h$. We answer the following question: Under what circumstances must $h k$ be selfadjoint given that it is normal?
\end{abstract}

Let $h, k \in \mathcal{L}(\mathcal{H})$ be two selfadjoint bounded linear operators on a Hilbert space $\mathcal{H}$ such that $h k$ is normal. The example $h:=\left(\begin{array}{ll}0 & 1 \\ 1 & 0\end{array}\right), \quad k:=\left(\begin{array}{cc}0 & i \\ -i & 0\end{array}\right)$, for which $h k=\left(\begin{array}{cc}-i & 0 \\ 0 & i\end{array}\right)$, shows that $h k$ need not be selfadjoint; note that here $\sigma(h)=\sigma(k)=\{-1,+1\}$.

However, if either $h$ or $k$ is positive, then $h k$ is normal if and only if it is selfadjoint; for example, when $k \geq 0$,

$$
\sigma(h k) \subseteq \sigma\left(k^{\frac{1}{2}} h k^{\frac{1}{2}}\right) \cup\{0\} \subseteq \mathbf{R},
$$

and a normal operator with real spectrum is selfadjoint. In fact, $\sigma(h k)=\sigma(k h)=$ $\sigma\left(k^{\frac{1}{2}} h k^{\frac{1}{2}}\right)$ when $h$ is selfadjoint and $k$ is positive (see [6]; we thank Prof. J. Zemánek for this reference).

We note further that, because $\overline{\sigma(h k)} \backslash\{0\}=\{\bar{w}: 0 \neq w \in \sigma(h k)\}=\sigma\left((h k)^{*}\right) \backslash$ $\{0\}=\sigma(k h) \backslash\{0\}=\sigma(h k) \backslash\{0\}$, the spectrum $\sigma(h k)$ is symmetric with respect to the real axis. Conversely, any compact subset $K \subset \mathbb{C}$ of the complex plane which is symmetric with respect to the real axis can occur as the spectrum $\sigma(h k)$ for such a pair of operators. To see this, choose a countable sequence $\left(\lambda_{n}\right)_{n \in \mathbb{N}}$ dense in $K$. Then the operators

$$
h:=\bigoplus_{n=1}^{\infty}\left(\begin{array}{ll}
0 & 1 \\
1 & 0
\end{array}\right) \text { and } k:=\bigoplus_{n=1}^{\infty}\left(\begin{array}{cc}
0 & \lambda_{n} \\
\bar{\lambda}_{n} & 0
\end{array}\right)
$$

are selfadjoint operators on a separable Hilbert space such that $h k$ is normal and $\sigma(h k)=K$.

The question naturally arises of finding conditions that will guarantee that $h k$ is selfadjoint given that it is normal; we shall answer it in the wider context of Banach algebras.

Received by the editors November 15, 1999

2000 Mathematics Subject Classification. Primary 46H99; Secondary 47B15, 47B40.

Key words and phrases. Hilbert space, operator, normal, selfadjoint, hermitian, numerical range, Banach algebra. 
In what follows $A$ will be a complex unital Banach algebra with unit $e$. Recall that an element $a \in A$ is hermitian if $\|\exp (i t a)\|=1(t \in \mathbf{R})$ and hermitianequivalent if $\sup _{t \in \mathbf{R}}\|\exp (i t a)\|<\infty$. Also recall that $n$ is normal (-equivalent) if $n=r+i s$ where $r s=s r$ and $r, s$ are hermitian (-equivalent); we write $n^{*}=r-i s$ for such an $n$.

We shall use an extended two-normal Fuglede theorem:

Theorem. Let $A$ be a complex unital Banach algebra. If $m, n(\in A)$ are normalequivalent and na $=$ am for some $a \in A$, then $n^{*} a=a m^{*}$.

Proof. This follows from the fact that for any complex number $\lambda$ we have $a=$ $e^{i \bar{\lambda} n} a e^{-i \bar{\lambda} m}$ so that $\lambda \mapsto e^{i \lambda n^{*}} a e^{-i \lambda m^{*}}=e^{i\left[\bar{\lambda} n+\lambda n^{*}\right]} a e^{-i\left[\bar{\lambda} m+\lambda m^{*}\right]}$ is bounded and analytic, hence constant. See also [2], Theorem 1.

We require the following lemma:

Lemma 1. Let $A$ be a complex unital Banach algebra, and let $s, k \in A$. Suppose that $k$ is hermitian-equivalent, that $\sigma(k) \cap \sigma(-k) \subseteq\{0\}$, and that $s k+k s=0$. Then $k s=0$.

Proof. Regard $k$ as an element of $\mathcal{L}(A)$. Since $k$ is of class $\left(C^{1}(\mathbf{R})\right)$ (1], Satz 3.3 ), it is decomposable ([4], Theorem 3.1.19). Since $s(-k)=k s$, it follows from (4], Theorem 2.3.3 and [2], Proposition 1) that $\operatorname{ran} s \subseteq X_{k}(\sigma(k)) \cap X_{k}(-\sigma(k))=$ $X_{k}(\{0\})=\operatorname{ker} k$, where $X_{k}(F)$ is the spectral maximal subspace of $k$ corresponding to the set $F$; so $k s=0$.

Remark. In the particular case that $A$ is a $C^{*}$-algebra we can use the continuous functional calculus to give a different proof of this lemma. Since the map $\operatorname{sr}_{k}$ : $\sigma\left(k^{2}\right) \rightarrow \sigma(k): \lambda^{2} \mapsto \lambda$ is continuous, it follows that $k$ belongs to the bicommutant of $k^{2}$. Then $s k^{2}-k^{2} s=(s k+k s) k-k(s k+k s)=0$; hence $s k=k s$; hence $k s=0$.

Remark. When $A$ is a general Banach algebra and $\sigma(k) \cap \sigma(-k)=\emptyset$, we can use the holomorphic functional calculus (as in the previous Remark) to obtain the result of Lemma 1, without calling on the theory of generalized spectral operators.

The principal result of this paper is:

Theorem 1. Let $A$ be a complex unital Banach algebra. Suppose that $h, k(\in A)$ are hermitian-equivalent, that $\sigma(k) \cap \sigma(-k) \subseteq\{0\}$, and that $r=\frac{1}{2}(h k+k h)$ and $s=\frac{1}{2 i}(h k-k h)$ are two commuting hermitian-equivalent elements of $A$. Then $h k$ is hermitian-equivalent and $h k=k h$.

Proof. Let $n=h k=r+i s$; then $n$ is normal-equivalent, $n^{*}=k h=r-i s$, and $n n^{*}=n^{*} n$. The two ways of grouping the product $k h k$ give $n^{*} k=k n$. Then $n k=k n^{*}$ by the Fuglede theorem, and subtracting gives $s k=-k s$. Lemma 1 now shows that $k s=0$, so that $0=h k s=r s+i s^{2}$. It follows from the spectral mapping theorem for the pair $(r, s)$ that $\sigma(s)=\{0\}$, from which $s=0$. Then $h k=k h=r$, which is hermitian-equivalent.

The result promised in the title is an immediate corollary of Theorem 1.

Theorem 2. Let $h, k \in \mathcal{L}(\mathcal{H})$ be selfadjoint and suppose that $\sigma(k) \cap \sigma(-k) \subseteq\{0\}$. If $h k$ is normal, then $h k=k h$, so hk is selfadjoint. 
Another corollary is a generalisation of a result of Radjavi and Rosenthal [7] (which also appears as [5], Problem 193).

Theorem 3. Let $A$ be a complex unital Banach algebra in which every element is normal-equivalent and in which $(h k)^{*}=k h$ for every pair of hermitian-equivalent elements $h, k$. Then $A$ is commutative, and may be renormed to be a $C^{*}$-algebra.

Proof. Let $H$ denote the set of hermitian-equivalent elements in $A$. In the first place $A=H+i H$. Secondly, if $h, k \in H$, then $h, 2\|k\|+k$ satisfy the hypotheses of Theorem 1 [and $\sigma(2\|k\|+k) \cap \sigma(-2\|k\|-k)=\emptyset]$. Hence $h k=k h$; so $A$ is commutative. We can now renorm [first renorm $A$ as a Banach space, then as a Banach subalgebra of $\mathcal{L}(A)$ ] so that all the elements of $H$ are simultaneously hermitian; by the Vidav-Palmer theorem $A$ is a $C^{*}$-algebra.

This problem was raised, and the solution found, during the Banach Algebras 1999 Conference. The authors are glad to express their gratitude to the Organisers of the Conference and to the Institutions which funded it for inviting us and providing excellent facilities and support.

\section{REFERENCES}

1. E. Albrecht, Funktionalkalküle in mehreren Veränderlichen für stetige lineare Operatoren auf Banachräumen, Man. Math. 14 (1974), 1-40. MR 50:5507

2. E. Albrecht, On some classes of generalized spectral operators, Archiv der Mathematik 30 (1978), 297-303. MR 57:10486

3. F.F. Bonsall and J. Duncan, Complete Normed Algebras, Springer, 1973. MR 54:11013

4. I. Colojoară and C. Foiaş, Theory of generalized spectral operators, Gordon and Breach, 1968. MR 52:15085

5. P.R. Halmos, Hilbert Space Problem Book (Second Edition), Springer Verlag, 1982. MR 84e:47001

6. M. Hladnik and M. Omladič, Spectrum of the Product of Operators, Proc. American Math. Soc. 102 (1988), 300-302. MR 90a:47008

7. H. Radjavi and P. Rosenthal, On invariant subspaces and reflexive algebras, Amer. J. Math. 91 (1969), 683-692. MR 40:4796

Fachbereich 9 Mathematik, Universität des Saarlandes, Postfach 151150, 66041 SaArBRÜCKEN, GERMANY

E-mail address: ernstalb@math.uni-sb.de

Department of Mathematics, University of Glasgow, Glasgow G12 8QW, Scotland

E-mail address: pgs@maths.gla.ac.uk 